\title{
STRONG COMPLETENESS OF THE LAMBEK CALCULUS WITH RESPECT TO RELATIONAL SEMANTICS
}

\author{
SZABOLCS MIKULÁS \\ Mathematical Institute of the Hungarian Academy of Sciences \\ Budapest, Pf. 12\%, H-1364, Hungary \\ E-mail: H3762MIK@ELLA.HU,H3762MIK@HUELLA.BITNET
}

In [vB88], Johan van Benthem introduces Relational Semantics (RelSem for short), and states Soundness Theorem for Lambek Calculus (LC) w.r.t. RelSem. After doing this, he writes: "it would be very interesting to have the converse too", i.e., to have Completeness Theorem. The same question is in [vB91, p. 235]. In the following, we state Strong Completeness Theorems for different versions of LC.

First of all, let us define the language of LC. Given a denumerable set $P$ of primitive symbols, we let the set of formulae Form $\mathrm{LC}_{\mathrm{C}}$ be the smallest set containing

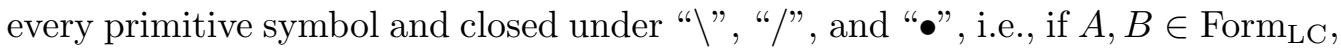
then $A \backslash B, A / B, A \bullet B \in$ Form $_{\mathrm{LC}}$. The set of sequents is the set of all expressions of the form $A_{1}, \ldots, A_{n} \Rightarrow A_{0}$ where $n$ is a positive integer and $A_{i} \in$ Form $_{\mathrm{LC}}$ for each $i \leq n$.

$\mathrm{LC}$ is given by the following axiom and rules of inference, where $A, B, C$ stand for formulae and $x, y, z$ stand for finite sequences of formulae including the empty sequence $\ominus$ unless the contrary is asserted.

Axiom:

$$
\text { (0) } A \Rightarrow A \text {. }
$$

1991 Mathematics Subject Classification: 03B65, 03G15, 68S05.

This is an extended version of the handout material prepared for (and distributed during) the lecture [Mi91] given at the Banach Center, 17th October, 1991.

Special thanks to Hajnal Andréka, thanks to Ildikó Sain and András Simon. Research supported by the Hungarian National Foundation for Scientific Research grants Nos. 1911 and 2258. 
Rules of inference:
$(1 \backslash) \frac{x \Rightarrow A \quad y, B, z \Rightarrow C}{y, x, A \backslash B, z \Rightarrow C} \quad x$ non-empty,
$(2 \backslash) \frac{A, x \Rightarrow B}{x \Rightarrow A \backslash B} \quad x$ non-empty,
(1/) $\frac{x \Rightarrow A \quad y, B, z \Rightarrow C}{y, B / A, x, z \Rightarrow C} \quad x$ non-empty,
$(2 /) \frac{x, A \Rightarrow B}{x \Rightarrow B / A} \quad x$ non-empty,
(3) $\frac{x \Rightarrow A \quad y \Rightarrow B}{x, y \Rightarrow A \bullet B} \quad x, y$ non-empty,
(4) $\frac{x, A, B, y \Rightarrow C}{x, A \bullet B, y \Rightarrow C}$,
(5) $\frac{x \Rightarrow A \quad A \Rightarrow B}{x \Rightarrow B} \quad x$ non-empty.

A theorem of $\mathrm{LC}$ is a sequent deducible in $\mathrm{LC}\left(\vdash_{\mathrm{LC}}\right)$, i.e., by the usual recursive definition, a sequent is a theorem iff it is an instance of $(0)$, or it is given by some rule of inference from some theorem(s). More generally, let $\Gamma$ be a set of sequents and $\varphi$ be a sequent. We say that $\varphi$ is LC-deducible from $\Gamma, \Gamma \vdash_{\mathrm{LC}} \varphi$, iff either

(i) $\varphi \in \Gamma$ or

(ii) $\varphi$ is an instance of (0) or

(iii) there is a set of sequents $\Delta$ each of whose elements is LC-deducible from $\Gamma$ and there is an inference rule such that $\frac{\Delta}{\varphi}$ is an instance of this rule.

R e mark. If the set of primitive symbols is the set of basic types, then the formulae are types and, roughly speaking, " $\Rightarrow$ " of LC corresponds to the derivability relation of Categorial Grammar. On the other hand, if $P$ is considered as a set of propositional variables, then LC is a Gentzen-type inference system, and hence it is a fragment of Linear Logic.

We give a Kripke-style semantics for LC, where we restrict the class of ordinary Kripke models with ternary accessibility relation to the class of models where the set of possible worlds consists of ordered pairs.

Definition of Relational Semantics. By a relational (Kripke) model for LC we mean an ordered triple $\langle W, C, v\rangle$ which is a Kripke model in the usual sense (i.e., $W$ is a set of possible worlds, $C$ is a (ternary) accessibility relation, and $v$ is an evaluation of expressions) and for which the following hold. $W$ is a transitive binary relation on some set $U$, and $C \subseteq W \times W \times W$ such that, for every $x, y, z \in W, C x y z$ holds iff $\{z\}=\{x\} \circ\{y\}$, i.e., iff there are $a, b, c \in U$ such that $x=\langle a, b\rangle, y=\langle b, c\rangle$ and $z=\langle a, c\rangle$. Moreover, let

$$
\operatorname{Exp} \stackrel{\text { def }}{=}\left\{A_{1}, \ldots, A_{n}: A_{i} \in \text { Form }_{\mathrm{LC}}, 1 \leq i \leq n, \text { for some } n\right\}
$$

$$
\cup\{\varphi: \varphi \text { is a sequent }\}
$$

and let $v: \operatorname{Exp} \rightarrow \mathcal{P}(W)$, the power set of $W$, be such that, for every $A, B \in$ Form $\mathrm{LC}_{\mathrm{C}}$, and for every sequence $x$ of formulae, $v(x, A)=v(x \bullet A)$ and

$$
\begin{aligned}
& v(A \bullet B) \stackrel{\text { def }}{=}\{z \in W:(\exists x \in v(A))(\exists y \in v(B)) C x y z\}, \\
& v(A \backslash B) \stackrel{\text { def }}{=}\{y \in W:(\forall x \in v(A)) \forall z(C x y z \rightarrow z \in v(B))\},
\end{aligned}
$$




$$
\begin{aligned}
v(B / A) & \stackrel{\text { def }}{=}\{x \in W:(\forall y \in v(A)) \forall z(C x y z \rightarrow z \in v(B))\}, \\
v(x \Rightarrow A) & \stackrel{\text { def }}{=}(W \backslash v(x)) \cup v(A) .
\end{aligned}
$$

We say that a sequent $\varphi$ of $\mathrm{LC}$ is true in a model $\langle W, C, v\rangle$, in symbols $\langle W, C, v\rangle$ $\vDash \varphi$, iff $v(\varphi)=W$, or, equivalently, the sequent $A_{1}, \ldots, A_{n} \Rightarrow A_{0}$ is true in the model above iff

$$
v\left(A_{1}\right) \circ \ldots \circ v\left(A_{n}\right) \subseteq v\left(A_{0}\right) .
$$

A formula is valid with respect to RelSem iff it is true in every model. We denote this by $\vDash_{\mathrm{R}} \varphi$. We say that $\varphi$ is a (RelSem) consequence of $\Gamma$, in symbols $\Gamma \vDash_{\mathrm{R}} \varphi$, iff, for every model $\langle\mathfrak{A}, v\rangle$, if $\langle\mathfrak{A}, v\rangle \vDash \Gamma$, then $\langle\mathfrak{A}, v\rangle \vDash \varphi$, where $\langle\mathfrak{A}, v\rangle \vDash \Gamma$ abbreviates that, for every $\psi \in \Gamma,\langle\mathfrak{A}, v\rangle \vDash \psi$.

Re mark. In the above definition, Exp can be thought of as the set of formulae of a modal logic with three binary modalities: "•", "\", "/". Then $C$ is the accessibility relation corresponding to the possibility-type modality "•", and the residuations are, in a certain sense, dual modalities of "•". (Indeed, the modality " " is related to the modality "•" in a similar fashion as the temporal modality "Always in the past", denoted by "[P]", is related to "Sometime in the future", denoted by " $\langle F\rangle$ ", in, e.g., [ANS91] and [Go87]. In [ANS91], " $\langle P\rangle$ " is called the conjugate of " $\langle F\rangle$ " and " $[P]$ " is the dual of " $\langle P\rangle$ ". So "Y" is a dual of a conjugate of "•" $\left(-(A \backslash-B)=A^{-1} \bullet B\right)$. It is instructive to meditate over the two steps

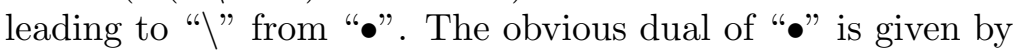

$$
v\left(A^{-1} \backslash B\right)=\{z:(\forall x \in v(A))(\exists y \in v(B)) C x y z\} .
$$

A conjugate of this is defined as

$$
v(A \backslash B)=\{z:(\forall x \in v(A))(\exists y \in v(B)) C x z y\} .
$$

Another conjugate of the same modality is defined by

$$
v(B / A)=\{z:(\forall x \in v(A))(\exists y \in v(B)) C z x y\} .)
$$

Further, " $\Rightarrow$ " is considered as classical implication. We can extend the relation $\langle W, C, v\rangle \vDash \psi$ for $\psi \in \operatorname{Exp}$ in the usual way, i.e., it holds iff $v(\psi)=W$.

Now, we can formulate our first completeness theorem (which was first presented in [Mi91]).

Theorem 0 (Strong Completeness Theorem for LC w.r.t. RelSem). For any set $\Gamma$ of sequents, and for any sequent $\varphi$,

$$
\Gamma \vdash_{\mathrm{LC}} \varphi \quad \text { iff } \quad \Gamma \vDash_{\mathrm{R}} \varphi .
$$

Remark. In the case of $\Gamma=\emptyset$, we have Weak Completeness Theorem w.r.t. RelSem.

Corollary 0 (Compactness Theorem). For any set $\Gamma$ of sequents and sequent $\varphi$, if $\Gamma \vDash_{\mathrm{R}} \varphi$, then there is a finite $\Delta \subseteq \Gamma$ such that $\Delta \vDash_{\mathrm{R}} \varphi$. 
Theorem 0 is a consequence of Theorems 1 and 2 below, but we need some definitions before formulating them.

Now we define the relations " $\leq_{\Gamma}$ " and " $\equiv_{\Gamma}$ " on Form $_{\mathrm{LC}}$, for any set $\Gamma$ of sequents. We let, for every $A, B \in$ Form $_{\mathrm{LC}}$,

$$
\begin{aligned}
& A \leq_{\Gamma} B \quad \text { iff } \quad \Gamma \vdash_{\mathrm{LC}} A \Rightarrow B \quad \text { and } \\
& A \equiv_{\Gamma} B \quad \text { iff } \quad\left(A \leq_{\Gamma} B \text { and } B \leq_{\Gamma} A\right) .
\end{aligned}
$$

Let $\mathfrak{T}$ be the formula algebra of LC, i.e.,

$$
\mathfrak{T} \stackrel{\text { def }}{=}\left\langle\text { Form }_{\mathrm{LC}}, \backslash, /, \bullet\right\rangle
$$

where "\", "/" and "•" denote the obvious operations on Form ${ }_{\mathrm{LC}}$.

Let $\mathfrak{L}_{\Gamma}$ be the factor structure of $\mathfrak{T}$ by " $\equiv_{\Gamma}$ ", i.e.,

$$
\mathfrak{L}_{\Gamma} \stackrel{\text { def }}{=}\left\langle L, \backslash, /, \bullet, \leq_{\Gamma}\right\rangle
$$

where $L$ consists of the equivalence classes, i.e., $L=\left\{\bar{A}: A \in\right.$ Form $\left._{\mathrm{LC}}\right\}$ where $\bar{A}=\left\{B: A \equiv_{\Gamma} B\right\}$, and $\bar{A} \backslash \bar{B}=\overline{A \backslash B}, \bar{A} / \bar{B}=\overline{A / B}, \bar{A} \bullet \bar{B}=\overline{A \bullet B}$ and $\bar{A} \leq_{\Gamma} \bar{B}$ iff $A \leq_{\Gamma} B$.

Definition of Relational Structure (RS).

$$
\mathfrak{A} \in \mathrm{RS} \quad \text { iff } \quad \mathfrak{A}=\langle A, \backslash, /, \bullet, \leq\rangle
$$

where $A$ is a non-empty set, " $"$ ", “/" and "•" are arbitrary binary operations on $A$, and " $\leq$ " is a binary relation on $A$.

Clearly, $\mathfrak{L}_{\Gamma} \in \mathrm{RS}$ for every set $\Gamma$ of sequents.

Let $\Sigma$ be the following set of formulae (in the first-order language with equality of RS) where $x, y, z, u$ are variables:

$$
\begin{array}{ll}
(\mathrm{A} 1) x \leq x, & \\
(\mathrm{~A} 2)(x \bullet y) \bullet z \leq x \bullet(y \bullet z), & (\mathrm{A} 3) x \bullet(y \bullet z) \leq(x \bullet y) \bullet z, \\
\text { (A4) } x \bullet(x \backslash y) \leq y, & \text { (A5) }(y / x) \bullet x \leq y, \\
(\mathrm{~A} 6) x \bullet y \leq z \rightarrow y \leq x \backslash z, & \text { (A7) } x \bullet y \leq z \rightarrow x \leq z / y, \\
\text { (A8) } x \leq y \wedge y \leq z \rightarrow x \leq z, & \\
\text { (A9) } x \leq y \wedge z \leq u \rightarrow x \bullet z \leq y \bullet u, & \\
\text { (A10) } x \leq y \wedge y \leq x \rightarrow x=y . &
\end{array}
$$

These axioms say that an $\mathfrak{A} \in \mathrm{RS}$ satisfying them is an ordered semigroup, where " $\leq$ " is the partial ordering, "•" is the semigroup operation, which is monotonic w.r.t. " $\leq$ ", and $x \backslash y$ is the greatest element such that $x \bullet(x \backslash y) \leq y$ and similarly for $y / x$.

Now we are ready to formulate the following.

TheOREM 1. For any set $\Gamma$ of sequents,

$$
\mathfrak{L}_{\Gamma} \vDash \Sigma
$$

where $\mathfrak{L}_{\Gamma}$ is the factor structure and $\Sigma$ is the set of formulae above. 
Definition of Representable Relational Structure (RRS).

$$
\mathfrak{A} \in \text { fullRRS iff } \mathfrak{A}=\langle A, \backslash, /, \circ, \subseteq\rangle
$$

where $A=\mathcal{P}(W)$, for some fixed transitive binary relation $W$, and the operations of $\mathfrak{A}$ are left and right residuations relativized to $W$ and relational composition, respectively, i.e., for any binary relations $a, b \subseteq W$,

$$
\begin{gathered}
a \backslash b \stackrel{\text { def }}{=}\{\langle x, y\rangle \in W: \forall z(\langle z, x\rangle \in a \rightarrow\langle z, y\rangle \in b)\}, \\
b / a \stackrel{\text { def }}{=}\{\langle x, y\rangle \in W: \forall z(\langle y, z\rangle \in a \rightarrow\langle x, z\rangle \in b)\}, \\
a \circ b \stackrel{\text { def }}{=}\{\langle x, y\rangle \in W: \exists z(\langle x, z\rangle \in a \wedge\langle z, y\rangle \in b)\} .
\end{gathered}
$$

Let RRS $=$ SfullRRS, i.e., RRS consists of the substructures of every fullRRS.

We say that a sequent $A_{1}, \ldots, A_{n} \Rightarrow A_{0}$ is true in a RRS $\mathfrak{A}$ under the valuation $v$, in symbols $\langle\mathfrak{A}, v\rangle \vDash A_{1}, \ldots, A_{n} \Rightarrow A_{0}$, iff

$$
v\left(A_{1}\right) \circ \ldots \circ v\left(A_{n}\right) \subseteq v\left(A_{0}\right)
$$

where $v\left(A_{i}\right)(i \leq n)$ is given by the natural extension of $v$ from $P$ to Form ${ }_{\mathrm{LC}}$, i.e., for any formulae $A, B$,

$$
v(A \backslash B)=v(A) \backslash v(B), \quad v(B / A)=v(B) / v(A), \quad v(A \bullet B)=v(A) \circ v(B) .
$$

In other words, $v$ is a homomorphism from the formula algebra given above into an $\mathfrak{A} \in$ RRS (here we disregard " $\subseteq$ ", of course).

The main reason why Theorem 0 holds is the following representation theorem, where IRRS denotes the collection of isomorphic copies of all elements of RRS.

THEOREM 2. For every $\mathfrak{A} \in \mathrm{RS}$,

$$
\mathfrak{A} \vDash \Sigma \quad \text { iff } \quad \mathfrak{A} \in \text { IRRS. }
$$

Sketch of proof of Theorem 0. Soundness is easy to check.

For the other direction we assume $\Gamma \nvdash_{\mathrm{LC}} A_{1}, \ldots, A_{n} \Rightarrow B$. Let $A=A_{1} \bullet$ $\ldots \bullet A_{n}$. Then $\Gamma \nvdash_{\mathrm{LC}} A \Rightarrow B$, i.e., $\bar{A} \not_{\Gamma} \bar{B}$ in $\mathfrak{L}_{\Gamma}$. By Theorems 1 and 2 , $\mathfrak{L}_{\Gamma}$ is in RRS. Let $v$ be a map such that $v(p)=\bar{p}$ for every $p \in P$. Then we have $\left\langle\mathfrak{L}_{\Gamma}, v\right\rangle \not \models A_{1}, \ldots, A_{n} \Rightarrow B$, so $\langle W, C, v\rangle \not \models A_{1}, \ldots, A_{n} \Rightarrow B$ for a (Kripke) model $\langle W, C, v\rangle$. Since $\Gamma$ is true in $\left\langle\mathfrak{L}_{\Gamma}, v\right\rangle$, it is so in $\langle W, C, v\rangle$. So we have $\Gamma \not \nvdash_{\mathrm{R}} A_{1}, \ldots, A_{n} \Rightarrow B$.

Remark. If, in the definition of Relational Semantics, we require that $W=$ $U \times U$ for some set $U$, then (Weak) Completeness Theorem fails. Indeed, the sequent $y \Rightarrow y \bullet(y \backslash y)$ is valid in those RRS"s in which $W$ has the form $U \times U$, because $v(y \backslash y) \supseteq \operatorname{Id} \cap(U \times U)$, i.e., the value of $y \backslash y$ contains the identity relation. On the other hand, let $W=\{\langle 0,0\rangle,\langle 0,1\rangle\}$ and consider $\mathcal{P}(W) \in$ RRS. Let $a=$ $\{\langle 0,1\rangle\}$. Then $a \backslash a=\{\langle 0,0\rangle,\langle 0,1\rangle\}$ and $a \circ(a \backslash a)=\emptyset$, so $a \not \leq a \circ(a \backslash a)$. Thus the sequent $y \Rightarrow y \bullet(y \backslash y)$ is not valid in RRS and, therefore, it is not derivable in LC. See also [Do90]. 
Theorems 9 and 10 investigate this $U \times U$-type semantics and state strong completeness of certain versions of LC w.r.t. it.

Let LCD be LC plus the following two rules:

$$
\text { (6) } \frac{x \Rightarrow A \quad x \Rightarrow B}{x \Rightarrow A \sqcap B}, \quad \text { (7) } \frac{x \Rightarrow A \sqcap B}{x \Rightarrow A \quad x \Rightarrow B} \text {. }
$$

Let $\Theta$ be $\Sigma$ plus the following two formulae:

$$
\text { (A11) } z \leq x \wedge z \leq y \rightarrow z \leq x \sqcap y, \quad(\mathrm{~A} 12) z \leq x \sqcap y \rightarrow z \leq x \wedge z \leq y .
$$
LCD,

THEOREM 3. For each sequent $\varphi$ and set $\Gamma$ of sequents of the language of

$$
\Gamma \vdash_{\mathrm{LCD}} \varphi \quad \text { iff } \quad \Gamma \vDash_{\mathrm{R}^{\prime}} \varphi
$$

where in the definition of the consequence relation " $\models_{\mathrm{R}^{\prime}}$ " we further require that $v(A \sqcap B)=v(A) \cap v(B)$.

COROLlary 1. If we add the axiom

$$
A, B \Rightarrow B \bullet A
$$

to LC (or to LCD), then we have Strong Completeness Theorem w.r.t. Symmetric RelSem, i.e., we consider only those models $\langle W, C, v\rangle$ for which $v(A \bullet B)=v(B \bullet A)$ for all formulae $A, B$. Moreover, we also have Compactness Theorem.

Remark. As in the case of Theorem 0, we also have Weak Completeness Theorem, i.e., a sequent $A_{1}, \ldots, A_{n} \Rightarrow B$ is derivable in the above version of LC iff $v\left(A_{1}\right) \circ \ldots \circ v\left(A_{n}\right) \subseteq v(B)$ for every symmetric representable relational structure and valuation $v$.

On the other hand, strong completeness fails if we add " $\sqcup$ " to the set of operations of LC (Theorem 5).

Definition. Let $\{\cup, \cap, \circ\} \subseteq M \subseteq\left\{\cup, \cap, \circ,-,{ }^{-1}, \emptyset, \mathrm{Id}, \backslash, /\right\}$. Then $\mathrm{R}(M)$ is the class of all algebras (isomorphic to ones) whose elements are binary relations and whose operations are the members of $M$.

THEOREM 4. $\mathrm{R}(M)$ is a quasi-variety which is not finitely axiomatizable.

Let $\mathrm{Qe}(\mathrm{R}(M))$ denote the class of all quasi-equations that hold in $\mathrm{R}(M)$.

COROllary 2. $\mathrm{Qe}(\mathrm{R}(M))$ is not axiomatizable by finitely many quasiequations.

TheOREm 5. The Relational Semantics with a set of connectives $M$ has no strongly complete and sound inference system.

Id e a of proof. Given a strongly complete inference system, one can translate it to a finite set of quasi-equations axiomatizing $\mathrm{Qe}(\mathrm{R}(M))$.

Now we claim that LC is not (weakly) complete w.r.t. language models (LM) and that there is no extension of $\mathrm{LC}$ which is sound w.r.t. $U \times U$-type Relational 
Semantics and is strongly complete w.r.t. LM. First, we recall the definition of language models from [vB91, p. 189].

Definition of Language Model. A family of languages is a set $\left\{L_{i}: i \in I\right\}$, where $L_{i}$ is a set of finite sequences (words) over a finite alphabet.

A language model is a family of languages enriched with the following operations:

$$
\begin{gathered}
L_{a} \bullet L_{b} \stackrel{\text { def }}{=}\left\{x y: x \in L_{a}, y \in L_{b}\right\}, \\
L_{a} \backslash L_{b} \stackrel{\text { def }}{=}\left\{x:\left(\forall y \in L_{a}\right) y x \in L_{b}\right\}, \\
L_{b} / L_{a} \stackrel{\text { def }}{=}\left\{x:\left(\forall y \in L_{a}\right) x y \in L_{b}\right\} .
\end{gathered}
$$

A sequent $A_{1}, \ldots, A_{n} \Rightarrow A_{0}$ is true in a language model if

$$
v\left(A_{1}\right) \bullet \ldots \bullet v\left(A_{n}\right) \subseteq v\left(A_{0}\right)
$$

where $v$ is the valuation function defined in the obvious way. The consequence relation " ${ }_{\mathrm{LM}}$ " is also the usual one.

THEOREM 6. LC is not (weakly) complete w.r.t. language models.

THEOREM 7. There is no calculus containing LC which is sound w.r.t. $U \times U$ type Relational Semantics and strongly complete w.r.t. language models.

COROLlary $3 . \mathrm{LC}^{0}$, the version of the Lambek Calculus where we admit sequents with empty antecedent, is not strongly complete w.r.t. LM.

Now we turn to investigating the connection between $U \times U$-type Relational Semantics and (various versions of) the Lambek Calculus. The main results are Theorems 9 and 10, but, as before, the piths of the proofs are two representation theorems (Theorems 8 and 11).

Let $\Sigma^{+}$be $\Sigma$ plus the following four formulae:

$$
\begin{array}{ll}
x \leq y \rightarrow z \leq z \bullet(x \backslash y), & x \leq y \rightarrow z \leq(x \backslash y) \bullet z, \\
x \leq y \rightarrow z \leq z \bullet(y / x), & x \leq y \rightarrow z \leq(y / x) \bullet z .
\end{array}
$$

Let $\mathrm{RRS}^{+}$be the class of those $\mathfrak{A} \in \mathrm{RRS}$ for which there is a $\mathfrak{B} \in$ fullRRS such that $B=\mathcal{P}(U \times U)$ for some set $U$, and $\mathfrak{A}$ is a substructure of $\mathfrak{B}$.

TheOREm 8 (Andréka-Mikulás). For every $\mathfrak{A} \in \mathrm{RS}$,

$$
\mathfrak{A} \vDash \Sigma^{+} \quad \text { iff } \quad \mathfrak{A} \in \text { IRRS }^{+} .
$$

Let $\mathrm{LC}^{+}$be $\mathrm{LC}$ plus the following four rules:

$$
\begin{array}{cc}
\frac{A \Rightarrow B}{C \Rightarrow C \bullet(A \backslash B)}, & \frac{A \Rightarrow B}{C \Rightarrow(A \backslash B) \bullet C}, \\
\frac{A \Rightarrow B}{C \Rightarrow C \bullet(B / A)}, & \quad \frac{A \Rightarrow B}{C \Rightarrow(B / A) \bullet C} .
\end{array}
$$


Let " $\models_{\mathrm{R}^{+}}$" be the semantic consequence relation which is determined by those relational Kripke models where $W=U \times U$. Then we have the following consequence of Theorem 8.

Theorem 9 (Andréka-Mikulás; Strong Completeness Theorem for $\mathrm{LC}^{+}$w.r.t. RelSem). For any set $\Gamma$ of sequents, and for any sequent $\varphi$,

$$
\Gamma \vdash_{\mathrm{LC}^{+}} \varphi \quad \text { iff } \quad \Gamma \vDash_{\mathrm{R}^{+}} \varphi \text {. }
$$

Now, we turn to showing that if we allow the empty sequence to be the antecedent of sequents in the Lambek Calculus, then it will become strongly complete w.r.t. $U \times U$-type Relational Semantics.

Let the language of $\mathrm{LC}^{0}$ be defined as that of $\mathrm{LC}$ except that we do not exclude the sequents $A_{1}, \ldots, A_{n} \Rightarrow A_{0}$ where $n=0$, i.e., we allow sequents with the empty sequence as antecedent. These sequents will be denoted by $\Rightarrow A_{0}$ or $\ominus \Rightarrow A_{0}$.

Let $\mathrm{LC}^{0}$ be given by the axiom (0) and rules $(1 \backslash)-(5)$ without any restriction, i.e., any sequence of sequents (denoted as $x, y$ or $z$ in the definition of LC) can be empty. Let " $\mathrm{R}_{\mathrm{R}^{+}}$" be as above. Then the following theorem holds.

Theorem 10 (Andréka-Mikulás; Strong Completeness Theorem for LC ${ }^{0}$ w.r.t. RelSem). Let $\Gamma \cup\{\varphi\}$ be a set of sequents in the language of $\mathrm{LC}^{0}$. Then

$$
\Gamma \vdash_{\mathrm{LC}^{0}} \varphi \quad \text { iff } \Gamma \vDash_{\mathrm{R}^{+}} \varphi \text {. }
$$

As before, the above completeness theorem is a consequence of an algebraic representation theorem (Theorem 11 below).

DEFINITION OF RRS ${ }^{0}$.

$$
\mathfrak{A} \in \text { fullRRS }^{0} \quad \text { iff } \quad \mathfrak{A}=\left\langle A, \backslash, /, \circ, \subseteq, \operatorname{Id}_{U}, \emptyset\right\rangle
$$

where $A=\mathcal{P}(U \times U)$ for some set $U$, and $\langle A, \backslash, /, \circ, \subseteq\rangle \in$ RRS. Further, $\operatorname{Id}_{U}=$ $\{\langle u, u\rangle: u \in U\}$, and the empty set, $\emptyset$, is considered as a binary relation.

Let $\operatorname{RRS}^{0}=$ SfullRRS $^{0}$.

DEFINITION OF RS ${ }^{0}$.

$$
\mathfrak{A} \in \mathrm{RS}^{0} \quad \text { iff } \quad \mathfrak{A}=\langle A, \backslash, /, \bullet, \leq, e, 0\rangle
$$

where $\langle A, \backslash, /, \bullet, \leq\rangle \in \mathrm{RS}$ and $e, 0 \in A$.

Let $\Sigma^{0}$ be $\Sigma$ plus the following formulae:

$$
e \bullet x=x \bullet e=x, \quad 0 \bullet x=x \bullet 0=0, \quad 0 \leq x .
$$

Let $\Delta$ be the set of the following formulae:

$$
\begin{aligned}
& x \bullet y=0 \leftrightarrow(x=0 \vee y=0), \\
& x \bullet y \leq e \leftrightarrow(x=0 \vee y=0 \vee x=y=e) .
\end{aligned}
$$

Note that $\Delta$ is not valid in $\operatorname{RRS}^{0}$ (while $\Sigma^{0}$ is). That is why, in the following theorem, only one direction is stated. 
TheOREm 11 (Andréka-Mikulás). For every $\mathfrak{A} \in \mathrm{RS}^{0}$,

if $\mathfrak{A} \vDash \Sigma^{0} \cup \Delta$, then $\mathfrak{A} \in \mathrm{IRRS}^{0}$.

\section{References}

[An88] H. Andréka, On the representation problem of distributive semilattice-ordered semigroups, preprint, Budapest 1988

[An91] - Representations of distributive lattice-ordered semigroups with binary relations, Algebra Universalis 28 (1991), 12-25.

[ANS91] H. Andréka, I. Németi and I. Sain, On the strength of temporal proofs, Theoret. Comput. Sci. 80 (1991), 125-151.

[vB88] J. van Benthem, Semantic parallels in natural language and computation, Institute for Language, Logic and Information, Univ. of Amsterdam, 1988; also appeared in: H.-D. Ebbinghaus et al. (eds.), Logic Colloquium (Granada 1987), North-Holland, 1989, 331-375

[vB91] —, Language in Action, North-Holland, 1991.

[Do90] K. Došen, A brief survey of frames for the Lambek calculus, Z. Math. Logik Grundlag. Math. 38 (1992), 179-187.

[Go87] R. Goldblatt, Logics of Time and Computation, CSLI Lecture Notes 7, Stanford Univ., 1987.

[La58] J. Lambek, The mathematics of sentence structure, Amer. Math. Monthly 65 (1958), 154-170.

[Mi91] Sz. Mikulás, The completeness of the Lambek Calculus w.r.t. Relational Semantics, lecture at the Banach Center, Warsaw, 17th October, 1991.

[Né91] I. Németi, Algebraizations of quantifier logics, an introductory overview, Studia Logica $50(3 / 4)(1991), 485-570$.

[Ro91] D. Roorda, Resource Logics: Proof-theoretical Investigations, Ph.D. thesis, Fac. Math. and Comput. Sci., Univ. of Amsterdam, 1991.

[Sa90] I. Sain, Beth's and Craig's properties via epimorphisms and amalgamation in algebraic logic, in: C. H. Bergman, R. D. Maddux and D. L. Pigozzi (eds.), Algebraic Logic and Universal Algebra in Computer Science, Lecture Notes in Comput. Sci. 425, Springer, 1990, 209-226 